\title{
EVALUATION OF FIREFIGHTER EXPOSURE TO WOOD SMOKE DURING TRAINING EXERCISES AT BURN HOUSES
}

\author{
Sujan Fernando ${ }^{1}$, Lorraine Shaw ${ }^{1}$, Don Shaw ${ }^{1}$, Michael Gallea ${ }^{1}$, Lori VandenEnden ${ }^{1}$, Ron \\ House $^{2}$, Dave K. Verma ${ }^{1}$, Philip Britz-McKibbin ${ }^{1 *}$ and Brian E. McCarry ${ }^{1}$ \\ ${ }^{1}$ McMaster University, Department of Chemistry and Chemical Biology, 1280 Main St. W., \\ Hamilton, ON, L8S 4M1 Canada \\ ${ }^{2}$ University of Toronto, 80 St. George St., Toronto, ON, M5S 3H6 Canada \\ *Corresponding Author: britz@mcmaster.ca
}

Supplemental Experimental, Supplemental Tables (Table S1, Table S2, Table S3) and Supplemental Figure (Figure S1, Figure S2)

Appendix 1: Firefighter Questionnaire: Smoke Exposure Study 


\section{Supplemental Experimental.}

\section{GC-MS Method for Analysis of Smoke Exposure in Air and Skin Wipe Sample Extracts.}

Analysis was carried out on an Agilent 6890GC coupled to an Agilent 5973MSD in electron ionization (EI) mode (70eV). Analysis was carried out on an Agilent J\&W DB-17ht column (30 $\mathrm{m} \times 0.25 \mathrm{~mm} \times 0.15 \mu \mathrm{m})$. The injector was set to $250^{\circ} \mathrm{C}$ and operated in splitless mode. A $1 \mu \mathrm{L}$ injection of the sample was made. The starting oven temperature was $50^{\circ} \mathrm{C}$ and increased to $300^{\circ} \mathrm{C}$ using a continuous gradient of $8^{\circ} \mathrm{C} / \mathrm{min}$. Temperature was held at $300^{\circ} \mathrm{C}$ for $15 \mathrm{~min}$ resulting in a total run time of $46 \mathrm{~min}$. The MSD was operated in selective ion monitoring (SIM) mode. The quantifying and qualifying ions for each of the target compounds are summarized below.

\begin{tabular}{|c|c|c|}
\hline Methoxyphenols & $\begin{array}{c}\text { Quan } \\
\text { Ion }\end{array}$ & $\begin{array}{c}\text { Qual } \\
\text { Ion }\end{array}$ \\
\hline Guaiacol & 166 & 196 \\
\hline MethylGuaiacol & 180 & 210 \\
\hline EthylGuaiacol & 194 & 224 \\
\hline Syringol & 196 & 226 \\
\hline PropylGuaiacol & 209 & 238 \\
\hline Eugenol & 206 & 236 \\
\hline Methylsyringol & 210 & 240 \\
\hline Isoeugenol & 206 & 236 \\
\hline Ethylsyringol & 224 & 254 \\
\hline Propylsyringol & 238 & 268 \\
\hline Acetovanillone & 223 & 238 \\
\hline Guaiacylacetone & 209 & 252 \\
\hline Acetosyringone & 238 & 268 \\
\hline Butylsyringone & 253 & 282 \\
\hline Propylsyringone & 253 & 296 \\
\hline Synapelaldehyde & 222 & 280 \\
\hline
\end{tabular}

\begin{tabular}{|c|c|c|}
\hline PAHs & $\begin{array}{c}\text { Quan } \\
\text { Ion }\end{array}$ & $\begin{array}{c}\text { Qual } \\
\text { Ion }\end{array}$ \\
\hline Naphthalene & 128 & 102 \\
\hline 2-MethylNaphthalene & 142 & 115 \\
\hline 1-MethylNaphthalene & 142 & 115 \\
\hline Acenaphthylene & 152 & 124 \\
\hline Biphenyl & 154 & 128 \\
\hline Acenaphthene & 154 & 126 \\
\hline Fluorene & 166 & 139 \\
\hline Phenenthrene & 178 & 152 \\
\hline Fluoranthene & 202 & 101 \\
\hline Pyrene & 202 & 101 \\
\hline Benzo[ghi]fluoranthene & 226 & 113 \\
\hline Benzo[c]phenanthrene & 228 & 114 \\
\hline Cyclopenta[cd]pyrene & 226 & 113 \\
\hline Benz[a]anthracene & 228 & 114 \\
\hline Chrysene & 228 & 114 \\
\hline Benzo[b]fluoranthene & 252 & 126 \\
\hline Benzo[j]fluoranthene & 252 & 126 \\
\hline Benzo[k]fluoranthene & 252 & 126 \\
\hline Benzo[a]fluoranthene & 252 & 126 \\
\hline Benzo[e]pyrene & 252 & 126 \\
\hline Benzo[a]pyrene & 252 & 126 \\
\hline Perylene & 252 & 126 \\
\hline
\end{tabular}


GC-MS/MS Method for the Analysis of Smoke Exposure Markers in Urine. Due to the lower levels of smoke markers and their oxidized metabolites/conjugates and their isomers in urine, analysis was carried out on a Varian CP-3800GC coupled to a Varian 1200L triple quadrupole $\mathrm{MS}$ in EI mode $(70 \mathrm{eV})$. Analysis was carried out on an Agilent J\&W DB-17ht column $(30 \mathrm{~m} \times 0.25 \mathrm{~mm} \times 0.15 \mu \mathrm{m})$. The injector was set to $250^{\circ} \mathrm{C}$ and operated in splitless mode. A $1 \mu \mathrm{L}$ injection of the sample was made. The starting oven temperature was $40^{\circ} \mathrm{C}$ and increased to $300^{\circ} \mathrm{C}$ using a continuous gradient of $8^{\circ} \mathrm{C} / \mathrm{min}$. Temperature was held at $300^{\circ} \mathrm{C}$ for 2 min resulting in a total run time of $35 \mathrm{~min}$. The MS was operated in multiple reaction monitoring (MRM) mode. The collision gas used was argon at a collision cell pressure of 1.5 torr. The MRM transitions for the target compounds are listed below.

\begin{tabular}{|c|c|c|}
\hline Methoxyphenols & $\begin{array}{c}\text { MRM } \\
\text { Transition } \\
\end{array}$ & $\begin{array}{c}\text { Collision } \\
\text { Energy (V) }\end{array}$ \\
\hline Guaiacol & $196-->181$ & 10 \\
\hline Methylguaiacol & $210-->180$ & 10 \\
\hline Ethylguaiacol & $224-->209$ & 10 \\
\hline Syringol & $226-->211$ & 10 \\
\hline Eugenol & $236-->206$ & 10 \\
\hline Isoeugenol & $236-->206$ & 10 \\
\hline Propylguaiacol & $238-->223$ & 15 \\
\hline Acetovanillone & $238-->223$ & 10 \\
\hline Methylsyringol & $240-->210$ & 10 \\
\hline Ethylsyringol & $254-->239$ & 10 \\
\hline Propylsyringol & $268-->239$ & 15 \\
\hline Guaiacylacetone & $252-->209$ & 10 \\
\hline Acetosyringone & $268-->253$ & 15 \\
\hline Butylsyringone & $282-->267$ & 15 \\
\hline Propylsyringone & $296-->281$ & 10 \\
\hline Sinapylaldehyde & $280-->265$ & 10 \\
\hline \multicolumn{3}{|l|}{ Hydroxy-PAHs } \\
\hline 1-OH Naphthalene & $216-->201$ & 10 \\
\hline 2-OH Naphthalene & $216-->201$ & 10 \\
\hline 9-OH Fluorene & $254-->165$ & 15 \\
\hline 3-OH Fluorene & $254-->165$ & 15 \\
\hline 2-OH Fluorene & $254-->165$ & 15 \\
\hline 4-OH Phenanthrene & $266-->235$ & 25 \\
\hline 3-OH Phenanthrene & $266-->235$ & 25 \\
\hline 2-OH Phenanthrene & $266-->235$ & 25 \\
\hline 3-OH Fluoranthene & $290-->259$ & 30 \\
\hline 1-OH Pyrene & $290-->259$ & 30 \\
\hline
\end{tabular}


LC-MS Method for the Analysis of Creatinine in Urine Samples. Due to the large variations in hydration status among firefighters, creatinine was used to normalize the concentration levels for all smoke marker compounds measured in urine. Analysis was carried out on a Waters 2690 HPLC coupled to a Waters Quattro Ultima triple quadrupole mass spectrometer. Analysis was carried out on a Kinetex 2.6u XBC18 column (50 x $2.1 \mathrm{~mm})$. Mobile phase used was a 50:50 water and acetonitrile containing $0.1 \%$ formic acid at a flow rate of $0.2 \mathrm{~mL} / \mathrm{min}$ with an isocratic elution for $5 \mathrm{~min}$. Urine samples were diluted by 1000 -fold in mobile phase and a $2 \mu \mathrm{L}$ injection was made. Prior to injection, each sample was spiked with creatinine- $\mathrm{d}_{3}$ and quantification was carried out using internal calibration method. A five-point calibration curve was made ranging in concentration from 0.1 to $10 \mu \mathrm{g} / \mathrm{mL}$. MRM was carried out for the creatinine $(114 \rightarrow 86)$ as well as creatinine- $d_{3}(117 \rightarrow 89)$. Argon was used as the collision gas with a collisional energy for precursors ions set at $20 \mathrm{~V}$. 


\section{Supplemental Tables.}

TABLE S1. The average airborne concentration of methoxyphenols (16 compounds) and PAHs (22 compounds) from five sampling sessions at four different burn houses/sites across Ontario.

\begin{tabular}{|c|c|c|c|c|c|c|c|c|c|c|}
\hline \multicolumn{11}{|c|}{ Average Airborne Concentrations $\left(\mu \mathrm{g} / \mathrm{m}^{3}\right)$ and Field Blanks (BLK) from Burn Houses } \\
\hline$M P s$ & $\begin{array}{l}A F D \\
(n=5)\end{array}$ & $B L K$ & $\begin{array}{l}B F D \\
(n=5)\end{array}$ & $B L K$ & $\begin{array}{l}C F D \\
(n=8)\end{array}$ & $B L K$ & $\begin{array}{c}D F D \\
(n=5)\end{array}$ & $B L K$ & $\begin{array}{c}E F D \\
(n=3)\end{array}$ & $B L K$ \\
\hline Guaiacol & $80 \pm 16$ & 9.4 & $29 \pm 13$ & 0.3 & $429 \pm 300$ & 1.2 & $185 \pm 86$ & 0.6 & $479 \pm 77$ & 2.5 \\
\hline Methylguaiacol & $65 \pm 21$ & 2.7 & $20 \pm 12$ & 0.7 & $121 \pm 86$ & 0.8 & $109 \pm 58$ & 1.7 & $408 \pm 62$ & 1.0 \\
\hline Ethylguaiacol & $56 \pm 21$ & 0.6 & $11.3 \pm 7.5$ & 0.1 & $174 \pm 120$ & 0.1 & $89 \pm 47$ & $n d$ & $332 \pm 69$ & 0.4 \\
\hline Syringol & $72 \pm 36$ & $n d$ & $11.9 \pm 9.9$ & nd & $513 \pm 500$ & 0.3 & $199 \pm 190$ & $n d$ & $568 \pm 350$ & nd \\
\hline Eugenol & $13.6 \pm 4.3$ & $n d$ & $2.8 \pm 1.8$ & $n d$ & $29 \pm 21$ & $n d$ & $18.6 \pm 8.6$ & $n d$ & $96 \pm 25$ & $n d$ \\
\hline Isoeugenol & $36 \pm 22$ & nd & $3.9 \pm 2.8$ & $n d$ & $97 \pm 120$ & $n d$ & $77 \pm 45$ & $n d$ & $335 \pm 150$ & $n d$ \\
\hline Propylguaiacol & $12.9 \pm 4.4$ & $n d$ & $2.2 \pm 1.5$ & $n d$ & $23 \pm 18$ & $n d$ & $21 \pm 15$ & $n d$ & $93 \pm 36$ & $n d$ \\
\hline Acetovanillone & $7.3 \pm 7.6$ & $n d$ & $2.7 \pm 1.0$ & $n d$ & $15 \pm 14$ & $n d$ & $23 \pm 17$ & $n d$ & $172 \pm 68$ & $n d$ \\
\hline Methylsyringol & $36 \pm 19$ & nd & $8.4 \pm 7.7$ & $n d$ & $164 \pm 170$ & $n d$ & $104 \pm 100$ & $n d$ & $145 \pm 91$ & $n d$ \\
\hline Ethylsyringol & $33 \pm 31$ & $n d$ & $3.6 \pm 2.2$ & $n d$ & $104 \pm 100$ & nd & $165 \pm 160$ & $n d$ & $237 \pm 130$ & $n d$ \\
\hline Propylsyringol & $22 \pm 30$ & $n d$ & $1.9 \pm 1.3$ & $n d$ & $52 \pm 52$ & $n d$ & $43 \pm 46$ & $n d$ & $49 \pm 23$ & $n d$ \\
\hline Guaiacylacetone & $30 \pm 17$ & $n d$ & $9.2 \pm 5.1$ & $n d$ & $134 \pm 130$ & $n d$ & $56 \pm 45$ & $n d$ & $416 \pm 170$ & $n d$ \\
\hline Acetosyringone & $27 \pm 12$ & $n d$ & $9.7 \pm 2.0$ & $n d$ & $127 \pm 110$ & $n d$ & $133 \pm 55$ & $n d$ & $222 \pm 70$ & $n d$ \\
\hline Butylsyringone & $7.9 \pm 3.2$ & nd & $3.2 \pm 1.6$ & $n d$ & $38 \pm 28$ & nd & $28 \pm 25$ & $n d$ & nd & $n d$ \\
\hline Propylsyringone & nd & $n d$ & 0.3 & $n d$ & $8.1 \pm 5.0$ & nd & $2.4 \pm 1.2$ & $n d$ & $35 \pm 16$ & $n d$ \\
\hline Synapelaldehyde & 5.8 & $n d$ & nd & $n d$ & $4.0 \pm 0.6$ & $n d$ & $15 \pm 17$ & $n d$ & nd & $n d$ \\
\hline Total & 504 & & 120 & & 2032 & & 1268 & & 3587 & \\
\hline \multicolumn{11}{|l|}{$P A H s$} \\
\hline Naphthalene & $46 \pm 12$ & 0.9 & $38 \pm 13$ & 0.3 & $108 \pm 107$ & 0.9 & $110 \pm 26$ & 1.4 & $119 \pm 20$ & 1.5 \\
\hline 2-methylnaphthalene & $5.2 \pm 4.4$ & 2.3 & $6.6 \pm 2.8$ & 0.4 & $28 \pm 26$ & 0.5 & $27.4 \pm 8.1$ & 2.8 & $37.6 \pm 8.9$ & 0.1 \\
\hline 1-methylnaphthalene & $6.7 \pm 1.4$ & $n d$ & $4.5 \pm 1.5$ & 0.6 & $16 \pm 16$ & 0.7 & $10.0 \pm 6.3$ & 2.6 & $19.6 \pm 4.8$ & $n d$ \\
\hline Acenaphthylene & $11.1 \pm 3.2$ & 0.5 & $5.9 \pm 2.5$ & 0.1 & $32 \pm 31$ & 0.2 & $27.6 \pm 8.3$ & $n d$ & $33 \pm 11$ & 0.3 \\
\hline Biphenyl & $5.1 \pm 1.5$ & 0.2 & $4.1 \pm 1.6$ & nd & $12 \pm 12$ & 0.2 & $10.0 \pm 2.1$ & $n d$ & $14.6 \pm 5.6$ & 0.4 \\
\hline Acenaphthene & $2.6 \pm 1.5$ & nd & nd & $n d$ & $1.0 \pm 0.7$ & nd & $1.9 \pm 0.4$ & $n d$ & $2.7 \pm 2.7$ & $n d$ \\
\hline Fluorene & $4.2 \pm 1.4$ & 0.1 & $2.0 \pm 1.0$ & $n d$ & $15 \pm 12$ & $n d$ & $8.6 \pm 2.6$ & $n d$ & $18.7 \pm 6.1$ & $n d$ \\
\hline Phenanthrene & $16.9 \pm 7.3$ & 0.1 & $11.8 \pm 6.1$ & 0.1 & $45 \pm 47$ & 0.2 & $28.6 \pm 7.0$ & 0.3 & $76 \pm 34$ & $n d$ \\
\hline Fluoranthene & $8.4 \pm 3.4$ & $n d$ & $3.6 \pm 2.1$ & $n d$ & $26 \pm 24$ & $n d$ & $13.5 \pm 2.3$ & 0.1 & $47 \pm 17$ & $n d$ \\
\hline Pyrene & $9.9 \pm 3.9$ & nd & $4.2 \pm 2.4$ & $n d$ & $26 \pm 24$ & $n d$ & $14.1 \pm 2.1$ & $n d$ & $45 \pm 22$ & $n d$ \\
\hline Benzo[ghi]fluoranthene & $1.2 \pm 0.2$ & $n d$ & $0.5 \pm 0.2$ & $n d$ & $5.6 \pm 3.0$ & $n d$ & $2.2 \pm 0.3$ & $n d$ & $12.8 \pm 2.7$ & $n d$ \\
\hline Benzo[c]phenanthrene & $0.5 \pm 1.2$ & $n d$ & $0.2 \pm 0.1$ & $n d$ & $2.0 \pm 0.9$ & $n d$ & $0.8 \pm 0.2$ & $n d$ & $10.6 \pm 3.6$ & $n d$ \\
\hline Cyclopenta[cd]pyrene & $1.6 \pm 0.3$ & $n d$ & $0.2 \pm 0.1$ & $n d$ & $7.4 \pm 5.3$ & $n d$ & $5.3 \pm 0.8$ & $n d$ & $10.6 \pm 3.4$ & $n d$ \\
\hline Benz[a]anthracene & $1.9 \pm 0.7$ & $n d$ & $0.7 \pm 0.4$ & $n d$ & $13 \pm 15$ & $n d$ & $3.5 \pm 0.4$ & $n d$ & $17.5 \pm 7.8$ & $n d$ \\
\hline Chrysene & $2.2 \pm 0.8$ & $n d$ & $1.1 \pm 0.5$ & $n d$ & $7.5 \pm 5.1$ & $n d$ & $3.8 \pm 0.6$ & $n d$ & $21.9 \pm 7.8$ & $n d$ \\
\hline Benzo[b]fluoranthene & $0.8 \pm 0.2$ & nd & $0.3 \pm 0.2$ & $n d$ & $3.8 \pm 2.2$ & nd & $1.5 \pm 0.2$ & $n d$ & $9.7 \pm 3.4$ & $n d$ \\
\hline Benzo[j]fluoranthene & $0.5 \pm 0.2$ & $n d$ & $0.2 \pm 0.1$ & $n d$ & $2.7 \pm 1.8$ & nd & $1.3 \pm 0.2$ & $n d$ & $7.0 \pm 2.7$ & $n d$ \\
\hline Benzo[k]fluoranthene & $0.7 \pm 0.2$ & $n d$ & $0.3 \pm 0.1$ & $n d$ & $2.7 \pm 1.8$ & $n d$ & $1.3 \pm 0.2$ & $n d$ & $10.5 \pm 5.3$ & $n d$ \\
\hline Benzo[a]fluoranthene & $0.2 \pm 0.1$ & $n d$ & $0.1 \pm 1.2$ & $n d$ & $1.8 \pm 1.1$ & $n d$ & $0.5 \pm 0.1$ & $n d$ & $5.8 \pm 3.3$ & $n d$ \\
\hline Benzo[e]pyrene & $0.7 \pm 0.2$ & nd & $0.3 \pm 0.2$ & $n d$ & $2.3 \pm 1.7$ & $n d$ & $1.2 \pm 0.2$ & $n d$ & $8.9 \pm 4.8$ & $n d$ \\
\hline Benzo[a]pyrene & $1.0 \pm 0.4$ & $n d$ & $0.3 \pm 0.2$ & $n d$ & $4.9 \pm 3.0$ & $n d$ & $2.0 \pm 0.3$ & $n d$ & $14.3 \pm 7.3$ & $n d$ \\
\hline Perylene & $0.2 \pm 0.1$ & $n d$ & $0.1 \pm 1.0$ & $n d$ & $0.8 \pm 0.5$ & $n d$ & $0.4 \pm 0.1$ & $n d$ & $5.0 \pm 3.1$ & $n d$ \\
\hline Total & 128 & & 85 & & 364 & & 277 & & 548 & \\
\hline
\end{tabular}


TABLE S2. The average MP (15 compounds) and PAH (16 compounds) skin loadings (ng/cm²) measured for all subjects $(n=28)$ at five different burn houses used for firefighter training.

\begin{tabular}{|c|c|c|c|c|c|c|c|c|c|c|}
\hline Methoxyphenols & \multicolumn{2}{|c|}{ Wrist } & \multicolumn{2}{|c|}{ Neck } & \multicolumn{2}{|c|}{ Forehead } & \multicolumn{2}{|c|}{ Back } & \multicolumn{2}{|c|}{ Fingers } \\
\hline$\left(\mathrm{ng} / \mathrm{cm}^{2}\right)$ & Mean & Std Dev & Mean & Std Dev & Mean & Std Dev & Mean & Std Dev & Mean & Std Dev \\
\hline Guaiacol & 0.42 & 0.58 & 0.44 & 0.60 & 0.46 & 0.64 & 0.44 & 0.62 & 0.49 & 0.63 \\
\hline Methylguaiacol & 0.18 & 0.26 & 0.26 & 0.34 & 0.28 & 0.38 & 0.21 & 0.34 & 0.31 & 0.41 \\
\hline Ethylguaiacol & 0.29 & 0.23 & 0.38 & 0.29 & 0.37 & 0.26 & 0.31 & 0.27 & 0.72 & 0.63 \\
\hline Syringol & 0.45 & 0.41 & 0.63 & 0.51 & 0.47 & 0.37 & 0.46 & 0.40 & 1.64 & 2.24 \\
\hline Eugenol & 0.59 & 0.47 & 0.70 & 0.53 & 0.68 & 0.53 & 0.78 & 0.68 & 0.82 & 0.72 \\
\hline Isoeugenol & 0.02 & 0.03 & 0.08 & 0.14 & 0.02 & 0.02 & 0.02 & 0.04 & 0.13 & 0.26 \\
\hline Propylguaiacol & 0.05 & 0.13 & 0.07 & 0.13 & 0.07 & 0.15 & 0.06 & 0.12 & 0.09 & 0.15 \\
\hline Acetovanillone & 0.53 & 0.84 & 0.41 & 0.57 & 0.58 & 1.02 & 0.32 & 0.52 & 0.81 & 1.39 \\
\hline Methylsyringol & 0.11 & 0.12 & 0.28 & 0.59 & 0.09 & 0.10 & 0.06 & 0.07 & 0.64 & 0.84 \\
\hline Ethylsyringol & 1.50 & 2.04 & 1.87 & 2.54 & 2.31 & 3.81 & 2.07 & 3.21 & 2.96 & 3.40 \\
\hline Propylsyringol & 0.04 & 0.07 & 0.07 & 0.11 & 0.04 & 0.13 & 0.09 & 0.27 & 0.16 & 0.28 \\
\hline Guaiacylacetone & 0.61 & 1.05 & 0.62 & 0.47 & 0.64 & 0.57 & 0.46 & 0.43 & 1.48 & 2.14 \\
\hline Acetosyringone & 0.25 & 0.25 & 0.39 & 0.33 & 0.73 & 1.06 & 0.47 & 0.70 & 0.66 & 0.60 \\
\hline Butylsyringone & 0.32 & 0.25 & 0.35 & 0.28 & 0.45 & 0.36 & 0.35 & 0.29 & 0.50 & 0.53 \\
\hline Propylsyringone & 0.31 & 0.26 & 0.59 & 0.55 & 0.88 & 1.41 & 1.08 & 1.46 & 0.51 & 0.45 \\
\hline \multicolumn{11}{|l|}{ PAHs } \\
\hline Naphthalene & 1.33 & 2.11 & 1.11 & 1.82 & 1.26 & 1.68 & 1.39 & 2.24 & 1.19 & 1.17 \\
\hline 2-methylnaphthalene & 0.45 & 0.39 & 0.45 & 0.40 & 0.47 & 0.43 & 0.48 & 0.46 & 0.47 & 0.40 \\
\hline 1-methylnaphthalene & 0.15 & 0.14 & 0.16 & 0.14 & 0.17 & 0.16 & 0.19 & 0.17 & 0.17 & 0.19 \\
\hline Fluorene & 0.15 & 0.13 & 0.14 & 0.07 & 0.13 & 0.09 & 0.15 & 0.09 & 0.16 & 0.13 \\
\hline Phenenthrene & 1.03 & 0.61 & 0.99 & 0.51 & 1.04 & 0.72 & 1.00 & 0.64 & 1.16 & 0.84 \\
\hline Fluoranthene & 0.32 & 0.20 & 0.38 & 0.23 & 0.37 & 0.31 & 0.31 & 0.24 & 0.47 & 0.32 \\
\hline Pyrene & 0.22 & 0.16 & 0.34 & 0.27 & 0.30 & 0.27 & 0.23 & 0.22 & 0.41 & 0.33 \\
\hline Benz[a]anthracene & 0.19 & 0.22 & 0.30 & 0.35 & 0.42 & 0.60 & 0.31 & 0.44 & 0.26 & 0.22 \\
\hline Chrysene & 0.08 & 0.11 & 0.09 & 0.08 & 0.10 & 0.10 & 0.08 & 0.13 & 0.12 & 0.09 \\
\hline Benzo[b]fluoranthene & 0.07 & 0.11 & 0.05 & 0.05 & 0.05 & 0.06 & 0.03 & 0.07 & 0.07 & 0.07 \\
\hline Benzo[j]fluoranthene & 0.04 & 0.06 & 0.03 & 0.02 & 0.03 & 0.04 & 0.03 & 0.05 & 0.03 & 0.02 \\
\hline Benzo[k]fluoranthene & 0.05 & 0.09 & 0.07 & 0.08 & 0.10 & 0.13 & 0.10 & 0.15 & 0.05 & 0.05 \\
\hline Benzo[a]fluoranthene & 0.06 & 0.12 & 0.07 & 0.10 & 0.15 & 0.20 & 0.12 & 0.18 & 0.03 & 0.05 \\
\hline Benzo[e]pyrene & 0.06 & 0.09 & 0.07 & 0.06 & 0.12 & 0.14 & 0.08 & 0.11 & 0.06 & 0.05 \\
\hline Benzo[a]pyrene & 0.05 & 0.08 & 0.04 & 0.05 & 0.08 & 0.11 & 0.05 & 0.07 & 0.03 & 0.03 \\
\hline Perylene & 0.04 & 0.06 & 0.02 & 0.02 & 0.03 & 0.04 & 0.03 & 0.04 & 0.01 & 0.00 \\
\hline
\end{tabular}


TABLE S3. The pre-exposure and average post exposure concentrations of the selected urinary MP and OH-PAH markers measured for all firefighters $(n=28)$ at five different sites. All urine samples were normalized to creatinine using single-spot urine samples collected prior to exposure and $24 \mathrm{~h}$ urine post-exposure for each subject.

\begin{tabular}{|c|c|c|c|c|}
\hline \multirow{2}{*}{} & \multicolumn{3}{|c|}{ Concentration ( $\mu$ g/g Creatinine) } \\
\cline { 2 - 5 } & \multicolumn{2}{|c|}{ MP } & \multicolumn{2}{c|}{ OH-PAH } \\
\hline FF Code & Pre & Post (avg) & Pre & Post (Avg) \\
\hline AFD1 & 0.01 & 0.15 & 0.77 & 2.13 \\
\hline AFD2 & 0.00 & 0.14 & 0.90 & 2.68 \\
\hline AFD3 & 0.02 & 0.35 & 5.62 & 5.04 \\
\hline AFD4 & 0.02 & 0.78 & 1.25 & 2.07 \\
\hline AFD5 & 0.02 & 4.82 & 3.90 & 2.14 \\
\hline BFD1 & 15.93 & 5.37 & 4.54 & 5.65 \\
\hline BFD2 & 0.21 & 0.71 & 1.84 & 2.48 \\
\hline BFD3 & 0.60 & 1.03 & 0.90 & 1.60 \\
\hline BFD4 & 0.32 & 5.08 & 2.61 & 4.54 \\
\hline BFD5 & 4.26 & 5.45 & 9.78 & 3.52 \\
\hline CFD1 & 10.32 & 0.39 & 4.49 & 3.99 \\
\hline CFD2 & 0.08 & 1.66 & 1.38 & 3.13 \\
\hline CFD3 & 0.62 & 5.94 & 0.31 & 0.93 \\
\hline CFD4 & 1.19 & 2.32 & 1.09 & 4.91 \\
\hline CFD5 & 0.22 & 1.44 & 0.35 & 2.79 \\
\hline CFD6 & 0.15 & 0.52 & 1.07 & 4.02 \\
\hline CFD7 & 0.43 & 1.91 & 0.20 & 1.97 \\
\hline CFD8 & 1.88 & 5.70 & 1.83 & 6.94 \\
\hline DFD1 & 0.01 & 0.28 & 1.89 & 2.09 \\
\hline DFD2 & 0.31 & 2.00 & 7.04 & 8.38 \\
\hline DFD3 & 1.28 & 2.91 & 11.21 & 11.95 \\
\hline DFD4 & 0.54 & 4.94 & 1.64 & 1.75 \\
\hline DFD5 & 1.09 & 44.23 & 1.82 & 5.46 \\
\hline EFD1 & 2.37 & 1.25 & 0.29 & 1.83 \\
\hline EFD2 & 0.60 & 2.55 & 7.37 & 5.65 \\
\hline EFD3 & 7.48 & 3.88 & 11.12 & 15.51 \\
\hline EFD4 & 0.50 & 12.08 & 1.38 & 2.61 \\
\hline EFD5 & 0.22 & 12.29 & 1.62 & 28.43 \\
\hline & & & & \\
\hline
\end{tabular}




\section{Supplemental Figures.}

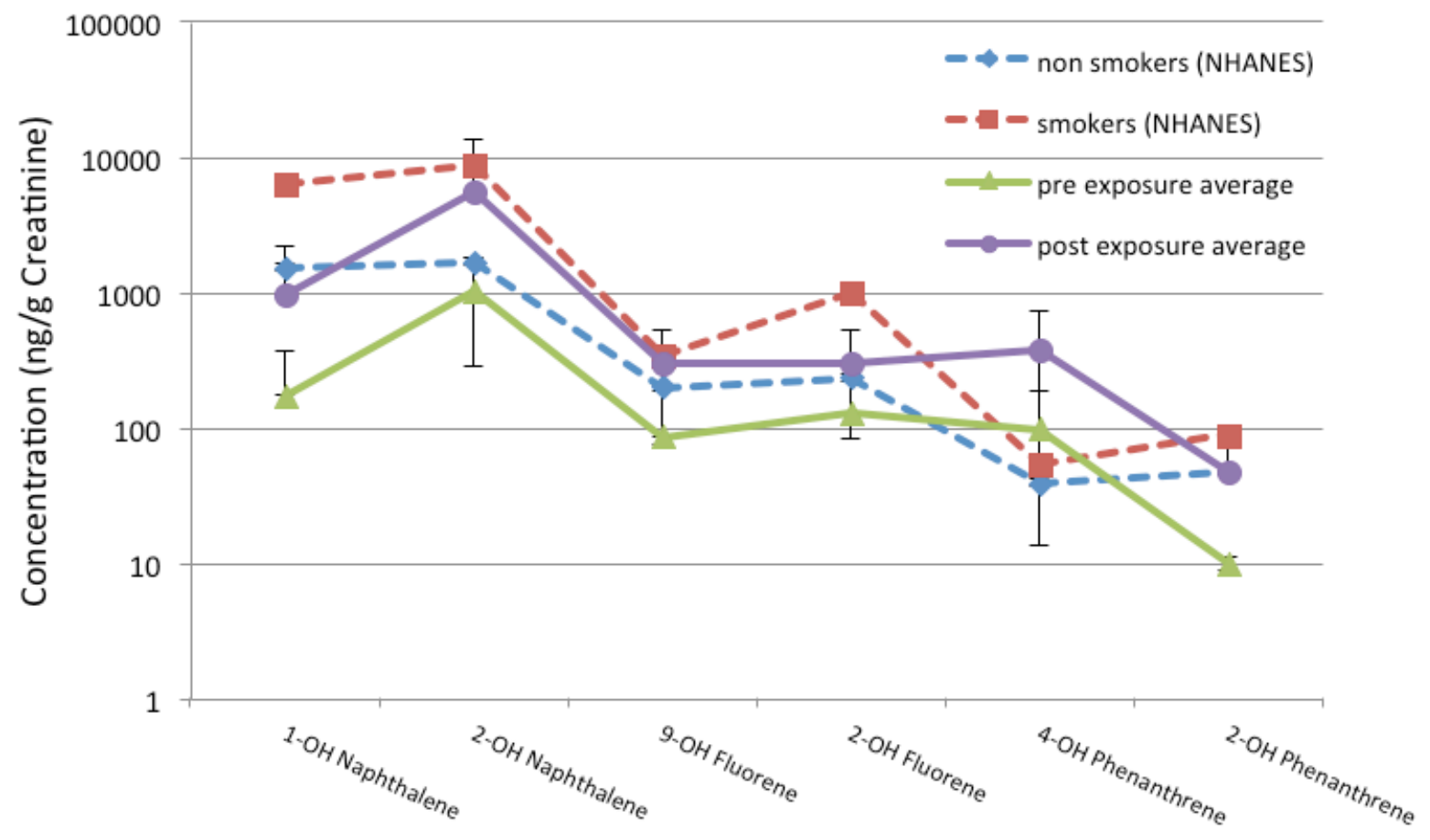

FIGURE S1. The urinary profile of representative hydroxy-PAH compounds from smokers and non-smokers in the US population determined as part of the NHANES study(18). Plotted alongside are the average pre-exposure and post-exposure profiles for 3 firefighters with the highest net increase in post-exposure samples from Figure $4 \mathrm{~b}$. This data highlights that the pre-exposure baseline is within the range of the non-smoker profile, whereas the post-exposure data is similar to median values measured for smokers suggestive of significant wood smoke exposure among nonsmoking firefighters equipped with bunker gear (i.e, personal protective equipment). 


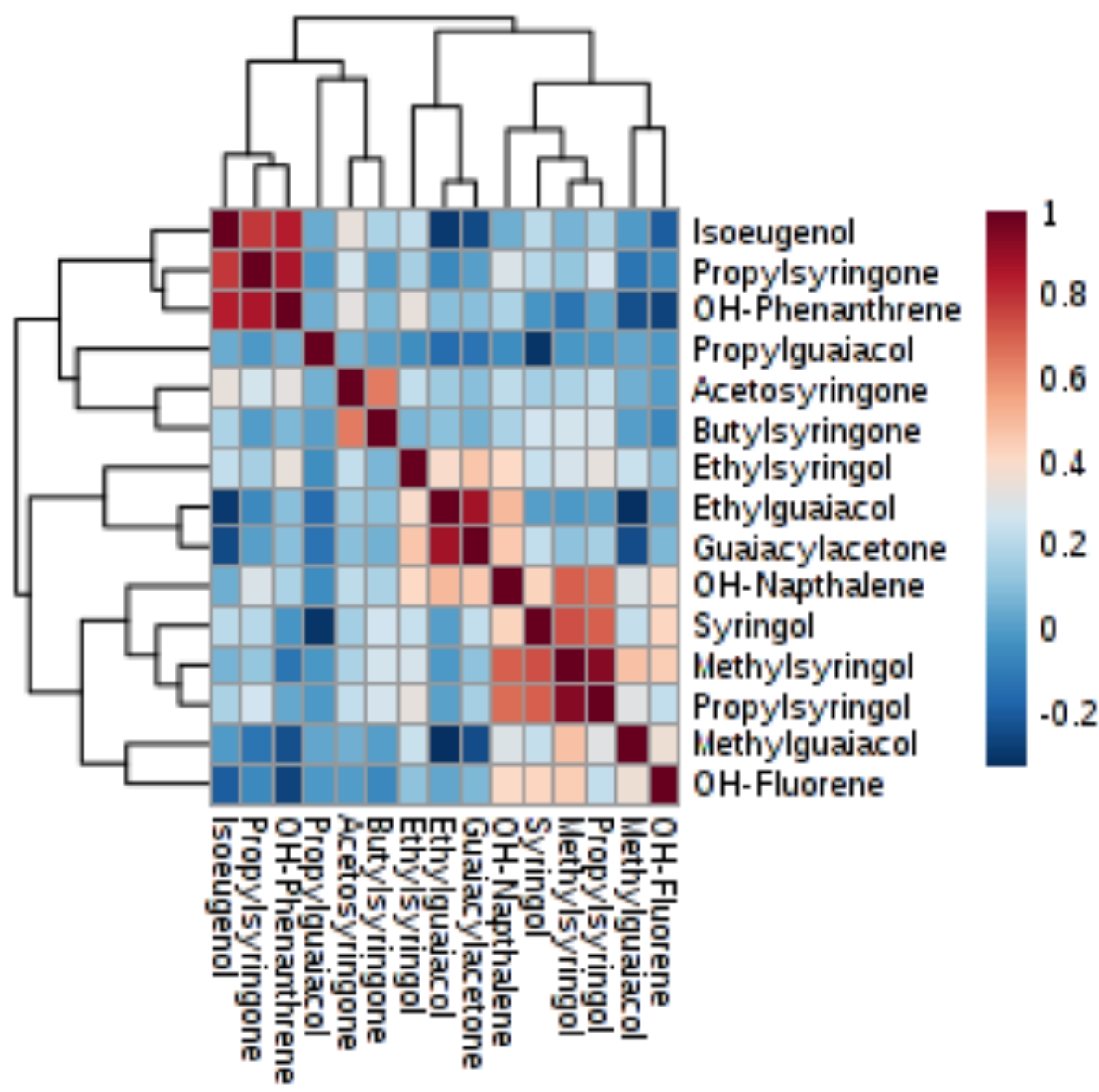

FIGURE S2. A correlation matrix highlighting the relationship between major MPs and (OH)PAHs consistently detected in air, skin and urine samples from firefighters following smoke exposure during exercise trials in burn houses. 


\section{Appendix 1. \\ FIREFIGHTER QUESTIONNAIRE: SMOKE EXPOSURE STUDY}

\section{Introduction:}

The purpose of this questionnaire is to determine the contributing factors pertaining to a fire fighter's overall chemical exposure to smoke. Please note that the information collected in this form is for the sole purpose of this research study supported by the Ministry of Labour (Ontario) - Bridging the Gap. It will not be disclosed, made available or shared unless Dr. Philip Britz-McKibbin, Department of Chemistry and Chemical Biology, McMaster University first obtains your written permission.

What method was used to complete this questionnaire (Please circle answer)? Self-Administered Interview

If by interview, provide name of interviewer and affiliation:

Interviewer Name:

Interviewer Affiliation:

1. Date Questionnaire Completed (dd-month-yy):

2. Name (optional):

3. What was your job title and job function during the live fire training exercise monitored by the McMaster University research team? 
4. Please provide a very simple sketch in the space below, or reverse side of this page, of where you were standing during the fire training exercise mentioned above. Please indicate where you were relative to the material being burned, including the approximate location of doors and windows.

5. Gender Male/Female

6. Age (years):

$18-25$

26-35

$36-45$

$>45$

7. Body Weight (lbs): 100-125

$126-150$

151-175

$176-200$

201-225

$>225$

8. Height (ft and inches): $<5^{\prime} 7^{\prime \prime}$

$5^{\prime} 7^{\prime \prime}-6^{\prime} 0^{\prime \prime}$

6'1"-6'6"

$>6^{\prime} 6^{\prime \prime}$

9. Do you smoke? Yes

No____ If yes, how many packs per week?

10. Have you ever smoked? If so, how many years? 
11. If "Yes" to question 9, how many years ago did you stop smoking?

12. How many days ago did you last eat any barbequed food?

13. Did you barbeque the food yourself?

14. Do you have a wood burning fireplace at home?

15. If you answered "Yes" to question 13, how many days ago did you use it?

16. Years of Service at this Fire Station:

17. Years of Service at Other Fire Station(s):

18. How many more years until you reach retirement/early retirement?

19. What were your previous occupations, if applicable?

\begin{tabular}{|c|c|c|}
\hline 1 & \#Years & Dates: \\
\hline$?$ & \#Years & Dates: \\
\hline & \#Years & Dates:_ \\
\hline 4 & \#Years & Dates: \\
\hline
\end{tabular}

20. Did you ever work as a volunteer Fire Fighter (circle one)? Yes No 
21. If "Yes", when (please provide dates)? What type of fires did you typically attend?

22. During your career as a fire fighter, which kinds of fires, listed below, have you attended (if you have an entry for "Other" please provide details)?

\begin{tabular}{|c|c|c|c|c|c|c|}
\hline Type of Fire & $\begin{array}{c}\text { Yes } \\
\text { (check) }\end{array}$ & $\begin{array}{c}\text { No } \\
\text { (check) }\end{array}$ & $\begin{array}{c}\text { Approx. } \\
\text { Number } \\
\text { or } \% \\
\text { of Total }\end{array}$ & $\begin{array}{l}\text { Typical \# of Fire } \\
\text { Engines/Personnel } \\
\text { Attending Fire }\end{array}$ & $\begin{array}{l}\text { Primary Visual and } \\
\text { Odour Components } \\
\text { Noted While at Fire, } \\
\text { either when in fire } \\
\text { AND while } \\
\text { standing outside } \\
\text { (eg. burning plastic } \\
\text { odour) }\end{array}$ & $\begin{array}{c}\text { Typical } \\
\text { Time } \\
\text { (hours) } \\
\text { Spent }\end{array}$ \\
\hline Car & & & & & & \\
\hline Chemical & & & & & & \\
\hline Forest & & & & & & \\
\hline $\begin{array}{l}\text { General } \\
\text { Industrial }\end{array}$ & & & & & & \\
\hline $\begin{array}{l}\text { Petrochemical } \\
\text { (i.e. Oil } \\
\text { Refinery, } \\
\text { filling station) }\end{array}$ & & & & & & \\
\hline Residential & & & & & & \\
\hline Other & & & & & & \\
\hline
\end{tabular}

Details of "Other" type of

fire: 
22. What events, other than fires, have you been called to (i.e. people trapped in homes, pets in trees, climbing accidents)? Please list an approximate percentage of time spent on such activities during your career to accompany such activities?

23. When going to a fire, please circle the personal protective equipment (PPE) worn Day Clothing Worn

Turnout Gear - Pants, Boots and Coat

SCBA

Helmet (with/without face shield)

Gloves

Other (Please specify)

24. When do you consider a fire to be effectively extinguished?

25. Once a fire has been effectively extinguished, what level of personal protection is worn during overhaul? 
26. With respect to maintenance of protective equipment used, how frequently is the equipment checked and overhauled?

27. Who is responsible for maintaining/cleaning bunker gear?

28. How frequently is your personal protective equipment replaced?

29. Do you ever notice any soot inside your PPE? If so where?

30. In your opinion how well does your PPE (i.e. Bunker gear, SCBA and other PPE apparel) protect you from combustion materials present at a fire? Do you feel that the PPE provides a protective seal between you and the environment in which you may be working? If not, where do you feel that it is inadequate? Can you comment on possible ways that the equipment could be improved? 
31. In your opinion, what factor(s) (ex. rain, snow, wind, temperature inversions, type of fire, time of arrival etc.) contribute most to the levels of toxic materials generated by a fire?

32. In your opinion, how do climatic conditions influence your ability to contain a fire?

33. What other activities do you engage in during your shift at the Fire Station, when NOT attending a fire? Please list the approximate number of hours or \% time spent per activity on the line beside the respective activity:

Cleaning/Maintaining Fire Station Vehicles:

Cooking:

Barbecue:

Watching TV:

Exercising:

Paperwork:

34. Do you have any hobbies/other activities that may involve products of combustion? Yes No

35. Once you return to the fire station, do you notice any characteristic odour(s) that you would associate with a specific type of fire before, during or after showering? Please list any that you have noticed below, along with a description of the odour. 
36. It has been suggested that, in some cases, following a fire and consequent

washing, individuals have observed soot on their pillow and around their elbows up to several days later. Is this a general occurrence? Have you made any additional observations regarding post fire exposure that you would like to contribute?

THANK YOU FOR TAKING THE TIME TO COMPLETE THIS QUESTIONNAIRE. THIS INFORMATION WILL BE USED TO BETTER UNDERSTAND FIRE FIGHTER EXPOSURE TO VARIOUS ITUATIONS THAT THEY MAY ENCOUNTER IN THEIR OCCUPATION. YOUR TIME IS MOST APPRECIATED!

PLEASE RETURN YOUR COMPLETED QUESTIONNAIRE TO:

\author{
Philip Britz-McKibbin \\ Department of Chemistry \& Chemical Biology \\ McMaster University \\ 1280 Main St. W., Hamilton, ON L8S 4M1
}

